\title{
First experience with the wearable cardioverter defibrillator in the Netherlands
}

\author{
R. E. Knops • K. M. Kooiman • J. N. ten Sande • J. R. de Groot • A. A. M. Wilde
}

Published online: 6 December 2011

(C) The Author(s) 2011. This article is published with open access at Springerlink.com

\begin{abstract}
The implantable cardioverter defibrillator (ICD) has significantly improved survival in patients with an increased risk of sudden cardiac death (SCD). The wearable cardioverter defibrillator (WCD) is an alternative to the ICD in patients with a transient ICD indication or those in whom an ICD temporarily cannot be implanted. We describe here the technical details of the WCD and report three patients who were treated with a WCD in an outpatient setting. The WCD allowed the cardiac condition of two patients to improve to such an extent that permanent ICD implantation was deemed unnecessary. This new form of therapy may result in significant cost reduction, avoidance of unnecessary ICD implantation, and increased patient satisfaction.
\end{abstract}

Keyword Wearable cardiac defibrillator

\section{Introduction}

The implantable cardioverter defibrillator (ICD) has significantly improved survival in patients with an increased risk of sudden cardiac death (SCD) $[1,2]$. Patients who are at risk of or have survived a life-threatening arrhythmia are eligible for ICD treatment according to the current guidelines $[3,4]$. However, pro-arrhythmic conditions may modify over time; in particular, the left ventricular ejection fraction (LVEF) may improve, resulting in a change in ICD indication. It might therefore take time before a

R. E. Knops $(\square) \cdot$ K. M. Kooiman · J. N. ten Sande •

J. R. de Groot • A. A. M. Wilde

Department of Cardiology, Academic Medical Center,

Meibergdreef 9, room B2-238,

1105 AZ Amsterdam, the Netherlands

e-mail: r.e.knops@amc.uva.nl definitive indication for ICD implantation can be established. On the other hand, if an ICD needs to be removed due to infection or technical problems, the patient's risk of SCD remains unaltered.

In these cases the wearable cardioverter defibrillator (WCD) can be used as a temporary protection for SCD. The WCD is a non-invasive alternative to an ICD and may serve as a bridge to recovery such that ICD implantation can be avoided or postponed.

The first clinical use of the WCD was described in 2000 [5]. It is effective in defibrillating life-threatening ventricular arrhythmias without bystander intervention [6]. We describe here the first use of the WCD in the Netherlands. In this contribution we report the technical details and clinical practice in three cases.

\section{Technical details}

The only WCD currently available in the Netherlands is the LifeVest 3,100 (LifeVest ${ }^{\circledR}$ Zoll-Lifecor, Pittsburgh, USA) [7]. The system consists of two main components: (1) an electrode belt and garment that surrounds the patient's chest, and (2) a monitor that the patient wears around the waist or from a shoulder strap, shown in Fig. 1. Washable garments are available in sizes to suit most patients. The WCD electrodes are dry and non-adhesive to the skin. The monitor weighs about $800 \mathrm{~g}$. The device contains pushbuttons and indicators for the user, and a speaker for alarms and voice prompts.

\section{Treatment sequence}

After detection of ventricular fibrillation (VF), the WCD has a response time of $25 \mathrm{~s}$ (programmable up to $55 \mathrm{~s}$ ) to allow 

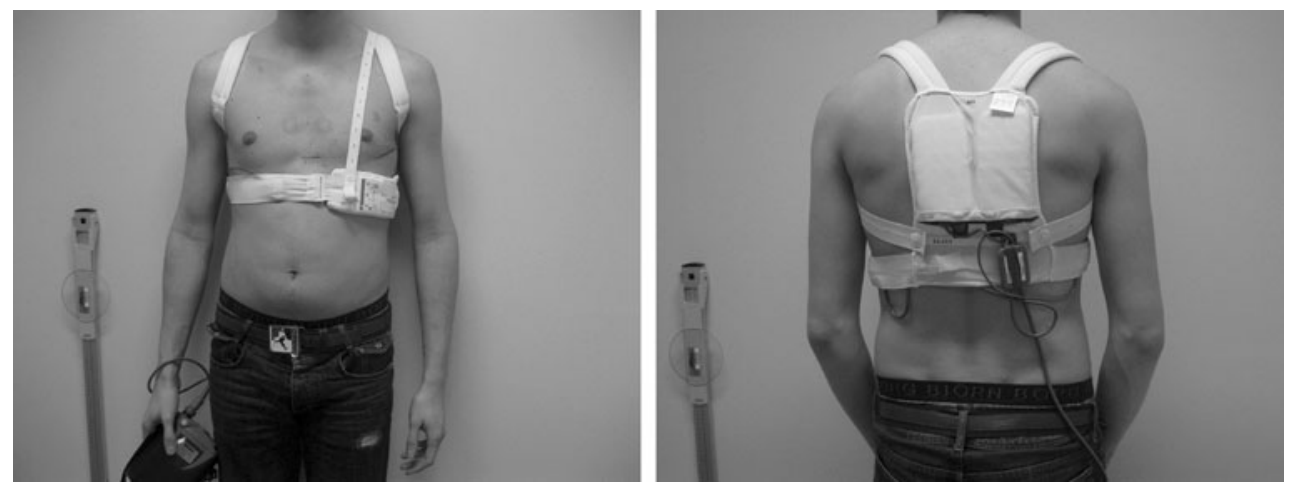

Fig. 1 Anterior and posterior view of LifeVest

the patient to react to the alarms, shown in Fig. 2. The threshold for VF identification can be set from 120 to 250 beats/min, with a default of 200 beats/min. If the system identifies VT, there is a response time of $60 \mathrm{~s}$ (programmable up to $180 \mathrm{~s}$ ). The threshold for VT identification can be programmed from 120 to the VF threshold, with a default setting of 150 beats/min. During normal operation, the vibration alarm activates before the siren alarm. If an arrhythmia is detected during the sleep interval (which can be programmed), the vibration and siren alarms activate simultaneously.

Directly prior to delivering a defibrillating shock, gel is released from the electrodes to lower skin impedance and yield optimal shock efficacy. The entire event, from arrhythmia detection to shock delivery, typically takes less than one minute. If the arrhythmia continues after the first shock, up to 5 additional shocks may be given. The shock energy can be programmed to between 75 and 150 joules $( \pm 5 \%)$, with a default setting of 150 joules.

Preventing inappropriate therapy

The key difference between the WCD and conventional ICDs is the opportunity given to the patient to interrupt the treatment cycle. When the alarm sounds as a result of arrhythmia detection, a conscious patient can stop the treatment. This prevents inappropriate arrhythmia detection from becoming inappropriate shocks. If the patient holds the two response buttons at any time during the treatment sequence, the alarm will stop and no shocks will be delivered. If the patient releases the response buttons, the device continues to give alarms, spoken warnings to bystanders, and ultimately delivers a shock.

\section{ECG recording of events}

The patient's ECG is stored for all detected arrhythmias. ECGs can also be recorded manually by pressing the response buttons on the device. Patients transmit device information telephonically to the LifeVest Network from where physicians can then access the data (ECG recordings, patient use, electrical interference, and other device-related information) through an encrypted internet connection.

\section{Clinical practice}

In our clinic we consider patients suitable for a WCD if they are at high risk for SCD and meet criteria for ICD implantation, but ICD implantation is precluded because of one of the following:

1. Primary prevention patients at high risk due to extremely low LVEF or therapy refractory nonsustained ventricular tachycardias (NSVT):
Fig. 2 Typical treatment sequence during $\mathrm{VF}$

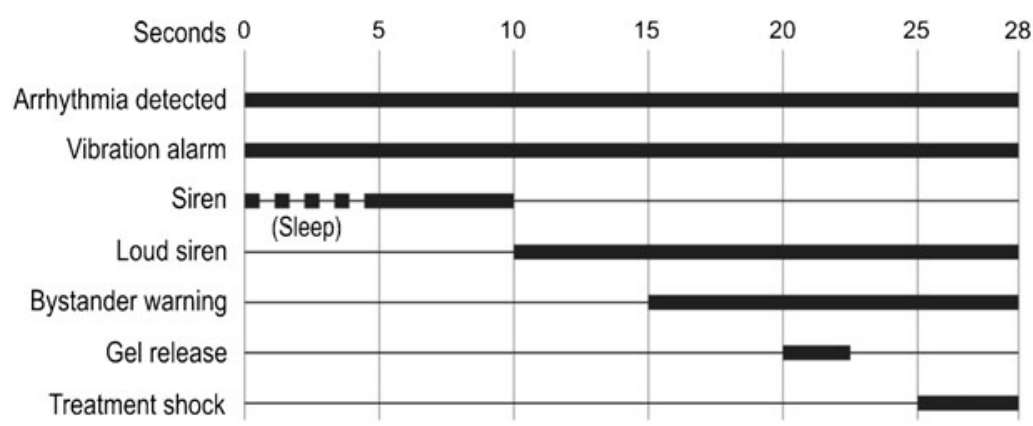


a. After recent MI (coverage during the 40 day ICD waiting period)

b. After recent CABG or PTCA (coverage during the 90 day ICD waiting period)

c. Listed for cardiac transplant

d. Recently diagnosed nonischaemic cardiomyopathy (to evaluate the result of optimal medical therapy)

2. Temporary medical condition precludes implantation (e.g. systemic infectious process)

3. Awaiting ICD re-implantation

We instruct eligible patients on the use and maintenance of the WCD. The patient is therefore admitted to the cardiology ward for at least $24 \mathrm{~h}$ to get comfortable with the device and the alarm signals.

An automatic external defibrillator (AED) is issued for occasions when the patient is not protected against SCD because of not wearing the WCD (e.g. during showering, garment changes). Partner, family and/or caretakers are instructed about AED use in these unprotected situations.

Outpatients are followed up 2 and 4 weeks after receiving the WCD, followed by monthly visits. During follow-up the arrhythmic events, patients' compliance and device use are evaluated. Also, a quality of life assessment is performed. An ICD technician and/or a cardiologist are available $24 \mathrm{~h}$ a day, 7 days a week for troubleshooting.

\section{Case reports}

We first started using the WCD in 2009 and describe three cases below.

\section{Case 1}

A 64-year-old woman was admitted to our clinic with dilated cardiomyopathy with a poor LVEF (19\% on MRI) resulting from viral myocarditis. A nonsustained ventricular tachycardia (NSVT) of 230 beats/min was observed. The low LVEF and NYHA class II-III constituted a class I indication for ICD implantation. However, LVEF might recover in patients with viral cardiomyopathy after optimal medical therapy for several months. Therefore, we postponed ICD implantation. The recording of an NSVT, however, indicated a high short-term risk for SCD. To allow her expected LVEF recovery in an outpatient setting we used the WCD in this patient for 3 months.

After a few days of wearing the WCD in our clinic the patient was discharged. Two manual events and one automatic event (noise) were recorded. There were no recorded VT/VF episodes. There was a very high compliance, but the patient decided not to use the WCD in the last week because of increasing wearing discomfort.
Three months after the start of WCD therapy her condition had improved significantly. Her LVEF increased to $39 \%$ and she was functionally in NYHA class I. Therefore, implantation of an ICD was no longer indicated.

Case 2

A 22-year-old male with a genetic predisposition to SCD due to the DPP6 haplotype was admitted with an acute Staphylococcus aureus pocket infection and sepsis after recent ICD implantation for primary prevention. Infection occurred after the patient manipulated the sutures of his wound.

The ICD was removed on the day of admission. The patient was admitted for several days for observation and intravenous antibiotic treatment. It was his specific request to have the new ICD implanted in the same position as the previous ICD. Our advice was to let the infected pocket recover for at least two months, and offered the patient the WCD to bridge this period in an outpatient setting.

The compliance of this patient was about $80 \%$ because of wearing discomfort and esthetical reasons. During the use of the WCD, 6 manual events and 20 automatic events were recorded All 20 automatic events were due to noise. There were no recorded episodes of VT/VF.

The infection had completely recovered after 2 months and a new ICD was implanted without complications in the same position. After 12 months of follow-up there were no signs of recurring infection.

\section{Case 3}

A 41-year-old male was referred to our clinic for ICD implantation as secondary prevention for idiopathic dilated cardiomyopathy and an episode of syncope. The patient had an excessive lifestyle and was familiar with abuse of alcohol, nicotine and intravenous drugs. Initial echocardiographic evaluation showed a dilated left ventricle and a poor LVEF. The coronary arteries were normal at angiography. The MRI confirmed a dilated cardiomyopathy with a poor left and right ventricular function, the calculated LVEF was $18 \%$. Taking into consideration the patient's lifestyle, we postulated that the cardiomyopathy might have resulted from alcohol toxicity.

No ventricular arrhythmias were recorded during admission. Medical treatment for heart failure was instituted, alcohol intake was prohibited and ICD implantation was deferred. We decided to bridge a period of 3 months with high risk of SCD with the WCD to allow the LVEF to improve.

During follow-up 1 manual event and 1 automatic event (noise) were recorded. There were no events of VT/VF. During the initial 2 months compliance was over $80 \%$, in 
the 3 rd month the compliance fell below $50 \%$ due to wearing discomfort.

After a period of three months the LVEF had fully recovered (EF: 60\%). Therefore, ICD implantation was no longer indicated.

\section{Conclusion}

We describe here the first clinical experience in the Netherlands with the WCD for the prevention of SCD. We could avoid unnecessary ICD implantation in two of our cases because of improvement of the patient's condition over time. In one case the WCD was used as a bridge to ICD re-implantation after device infection.

The cost of the WCD is considerable, and currently not reimbursed, but the costs only amount to approximately $20 \%$ of hospital admission in a telemetry monitored ward. For patients at increased risk for SCD, admission and telemetry is the only safe alternative for ICD implantation. Costs are further being saved when ICD implantation can be avoided, which was the case in two of the three patients that we describe. In addition to the cost issue, patients valued staying at home higher than hospital admission, evident from increased quality of life (not shown).

The safety of the WCD is a matter of ongoing debate. Feldman et al. report 289 patients of whom 6 patients who were not or incorrectly wearing the device died [6]. In their study 6 out of 8 appropriate shocks were successful. The two unsuccessful therapy attempts, one of which was nonfatal because of external defibrillation, both resulted from incorrect wearing of the device [6]. Meltendorf et al. demonstrated that 3 of the 4 VT/VF episodes were successfully terminated, and no patients experienced inappropriate WCD discharges [5]. These findings stress the importance of correct use of the WCD. This deserves attention, since our patients all experienced wearing discomfort, resulting from pressure sores from the electrodes, the weight of the monitor unit, the amount of false alarms and discomfort in the summer season. These issues resulted in different degrees of suboptimal protection for SCD.

This compliance-related suboptimal protection and the reports of patients who died not wearing the WCD [6] led us to think of a strategy to increase the safety of WCD therapy. It is virtually impossible to fit an unconscious patient with his/her WCD in an emergency setting. When therapy from a WCD is ineffective due to incorrect wearing it might be very difficult to correct this within a few minutes. For these situations we supply the patient's caretakers with an AED and instruct them on how to use this device. The AED serves as a backup device for the WCD. To our knowledge, we are the only group that attempts to protect the patient against SCD with an AED during the time that the WCD is not worn because of showering and garment changes. This strategy might increase the safety of this noninvasive alternative to ICD implantation.

The introduction of a new, smaller WCD (Zoll Medical LifeVest 4000) may potentially result in better patient compliance. Unfortunately this device is not yet available in the Netherlands.

We cannot draw conclusions on the efficacy of the WCD, since our patients did not experience appropriate therapy. It is of note, however, that no inappropriate shocks occurred either. This is congruent with Everitt's experience who reports no appropriate or inappropriate shocks with the WCD in children [8]. Feldman et al. describe an incidence of unnecessary shocks of $0.67 \%$ per month per patient use [6] which seems less than reported by Grimm et al. for the ICD [9].

It is likely that the use of the WCD in the Netherlands will further expand, and more knowledge will be acquired about patient compliance, effectiveness, usefulness and performance of the WCD. Klein et al. recently reviewed and summarised the experience with the WCD in 43 different hospitals in Germany [10]. They found that the WCD is effective in providing immediate lifesaving defibrillation, and that the device might contribute to better selection of patients for ICD therapy [10]. In their report, only $43 \%$ of WCD patients were eventually implanted with an ICD. This complements our findings.

Indeed, many patients who are implanted with an ICD never experience any appropriate therapy, even during longterm follow-up [11, 12]. This might underscore the need for a more accurate indication and a more detained attitude towards ICD implantation.

The WCD can be used to postpone or avoid ICD implantation in patients with a transient ICD indication or those in whom an ICD cannot be implanted temporarily. This new form of therapy may result in significant cost reduction (no long hospital stays), avoidance of unnecessary ICD implantation, and increased patient satisfaction. However, care needs to be taken to assure correct use of the device.

Open Access This article is distributed under the terms of the Creative Commons Attribution Noncommercial License which permits any noncommercial use, distribution, and reproduction in any medium, provided the original author(s) and source are credited.

\section{References}

1. Moss AJ, Zareba W, Hall WJ, et al. Prophylactic implantation of a defibrillator in patients with myocardial infarction and reduced ejection fraction. N Engl J Med. 2002;346:877-83.

2. The Antiarrhythmic Versus Implantable Defibrillators (AVID) Investigators. A comparison of antiarrhythmic-drug therapy with implantable defibrillators in patients resuscitated from 
near-fatal ventricular arrhythmias. N Engl J Med. 1997;337 (22):1576-83.

3. Zipes DP, Borggrefe M, Buxton AE, et al. ACC/AHA/ESC 2006 Guidelines for the prevention of sudden cardiac death. Europace. 2006;8(746):837.

4. Dickstein K, Vardas P, Auricchio A, et al. 2010 Focused update of ESC guidelines on device therapy in heart failure. Eur Heart J. 2010;31:2677-87.

5. Meltendorf U, Wollbrueck A, Auricchio A, et al. The wearable defibrillator - A new method to prevent sudden death. PACE. 2000;23(4 part II):606.

6. Feldman AM, Klein H, Tchou PJ, et al. Use of a wearable defibrillator in terminating tachyarrhythmias in patients at high risk of sudden death: results of the WEARIT/BIROAD. PACE. 2004;27(1):4-9.

7. Zoll Lifecor Cooperation. LifeVest System WCD 3100 Operator's Manual. 2009.
8. Everitt MD, Saarel EV. Use of the wearable defibrillator in children. PACE. 2010;33(6):742-6.

9. Grimm W, Menz V, Hoffmann J, et al. Complications of thirdgeneration implantable cardioverter-defibrillator therapy. PACE. 1999;22(1 part II):206-11.

10. Klein HU, Meltendorf U, Reek S, et al. Bridging a temporary high risk of sudden arrhythmic death. Experience with the wearable cardioverter defibrillator (WCD). PACE. 2010;33:353-67.

11. Mishkin JD, Saxonhouse SJ, Woo GW, et al. Appropriate evaluation and treatment of heart failurepatients after implantable cardioverterdefibrillator discharge: time to go beyond the initial shock. J Am Coll Cardiol. 2009;54(22):1993-2000.

12. Daubert JP, Zareba W, Cannom DS, et al. Inappropriate impantable cardioverter-defibrillator shocks in MADIT II. frequency, mechanisms, predictors, and survival impact. J Am Coll Cardiol. 2008;51 (1):357-65. 